ARTICLE

DOI: $10.1038 / s 41467-018-04446-8$

\title{
The hepcidin-ferroportin axis controls the iron content of Salmonella-containing vacuoles in macrophages
}

\author{
Daejin Lim¹,2, Kwang Soo Kim¹,2, Jae-Ho Jeong ${ }^{1,2}$, Oriana Marques $3,4,5$, Hyun-Ju kim ${ }^{1,2}$, Miryoung Song ${ }^{1,2}$, \\ Tae-Hoon Lee ${ }^{2,6}$, Jae $I I \mathrm{Kim}^{7,8}$, Hueng-Sik Choi ${ }^{9}$, Jung-Joon Min ${ }^{2,10}$, Dirk Bumann ${ }^{11}$, \\ Martina U. Muckenthaler ${ }^{3,4,5}$ \& Hyon E. Choy (D) 1,2
}

Macrophages release iron into the bloodstream via a membrane-bound iron export protein, ferroportin (FPN). The hepatic iron-regulatory hormone hepcidin controls FPN internalization and degradation in response to bacterial infection. Salmonella typhimurium can invade macrophages and proliferate in the Salmonella-containing vacuole (SCV). Hepcidin is reported to increase the mortality of Salmonella-infected animals by increasing the bacterial load in macrophages. Here we assess the iron levels and find that hepcidin increases iron content in the cytosol but decreases it in the SCV through FPN on the SCV membrane. Loss-of-FPN from the SCV via the action of hepcidin impairs the generation of bactericidal reactive oxygen species (ROS) as the iron content decreases. We conclude that FPN is required to provide sufficient iron to the SCV, where iron serves as a cofactor for the generation of antimicrobial ROS rather than as a nutrient for Salmonella.

\footnotetext{
${ }^{1}$ Department of Microbiology, Chonnam National University Medical School, Gwangju 61468, Republic of Korea. ${ }^{2}$ Department of Molecular Medicine (BK21plus), Chonnam National University Graduate School, Gwangju 61468, Republic of Korea. ${ }^{3}$ Department of Pediatric Hematology, Oncology and Immunology - University of Heidelberg, Im Neuenheimer Feld 350, Heidelberg D-69120, Germany. ${ }^{4}$ Molecular Medicine Partnership Unit, Heidelberg D-69120, Germany. ${ }^{5}$ Translational Lung Research Center Heidelberg (TLRC), German Center for Lung Research (DZL), University of Heidelberg, Heidelberg D-69120, Germany. ${ }^{6}$ Department of Biochemistry, Dental Science Research Institute, School of Dentistry, Chonnam National University and Korea mouse phenotype center (KMPC), Gwangju 61186, Republic of Korea. ${ }^{7}$ School of Life Sciences, Gwangju Institute of Science and Technology, Gwangju 61005, Republic of Korea. ${ }^{8}$ AnyGen, Gwangju Technopark, Gwangju 61008, Republic of Korea. ${ }^{9}$ National Creative Research Initiatives Center for Nuclear Receptor Signals and Hormone Research Center, School of Biological Sciences and Technology, Chonnam National University, Gwangju 61186, Republic of Korea. ${ }^{10}$ Department of Nuclear Medicine, Chonnam National University Medical School, Gwangju 61469, Republic of Korea. ${ }^{11}$ Focal Area Infection Biology, University of Basel, Basel CH-4056, Switzerland. These authors contributed equally: Daejin Lim, Kwang Soo Kim, Jae-Ho Jeong. Correspondence and requests for materials should be addressed to H.E.C. (email: hyonchoy@jnu.ac.kr)
} 
$\mathrm{n}$ response to infection, mammalian hosts deploy a strategy to withhold iron, an essential trace element for bacterial pro-

liferation ${ }^{1}$. This process is a central component of innate nutritional immunity controlled by the master iron regulatory hormone hepcidin, which is synthesized primarily in the liver in response to interleukin-6 (IL-6) signaling upon bacterial infection $^{2,3}$. Hepcidin promotes the degradation of its receptor, the sole known cellular iron exporter ferroportin (FPN), resulting in iron retention by macrophages and reduction of intestinal iron absorption $^{2}$. This mechanism decreases the serum iron content to about $30 \%$ of its normal level, a physiological change known as hypoferremia of infection ${ }^{4}$, which limits iron availability for extracellular bacteria but also leads to increased iron storage in macrophages of the liver and spleen. Previously, we reported that induction of hepcidin and eventual hypoferremia upon Salmonella infection was mediated by estrogen-related receptor (ERR) $\gamma^{5}$. An inverse agonist of ERR $\gamma$ (GSK5182) ameliorated Salmonella-mediated hypoferremia through reduction of hepcidin expression. Treatment of mice infected with Salmonella with GSK5182 resulted in an $\sim 30 \%$ decrease of iron in hepatic a

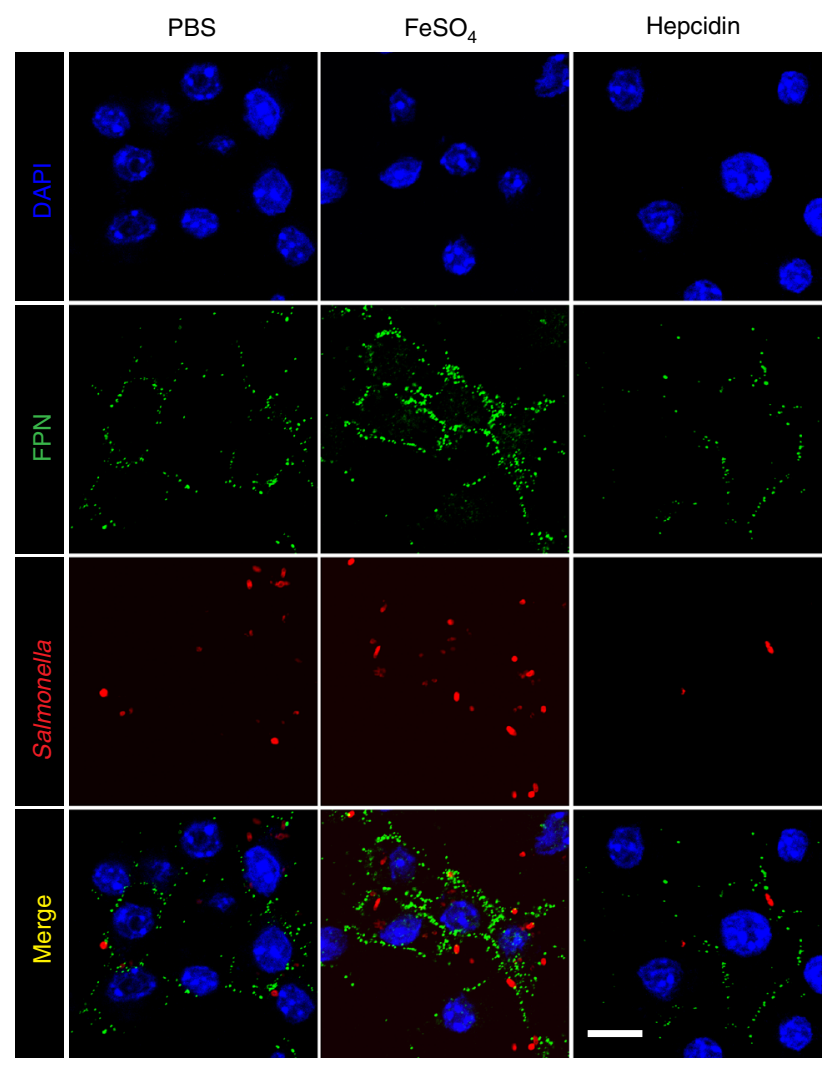

C

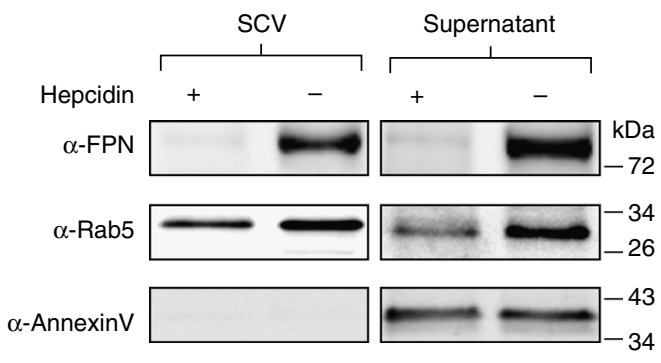

b

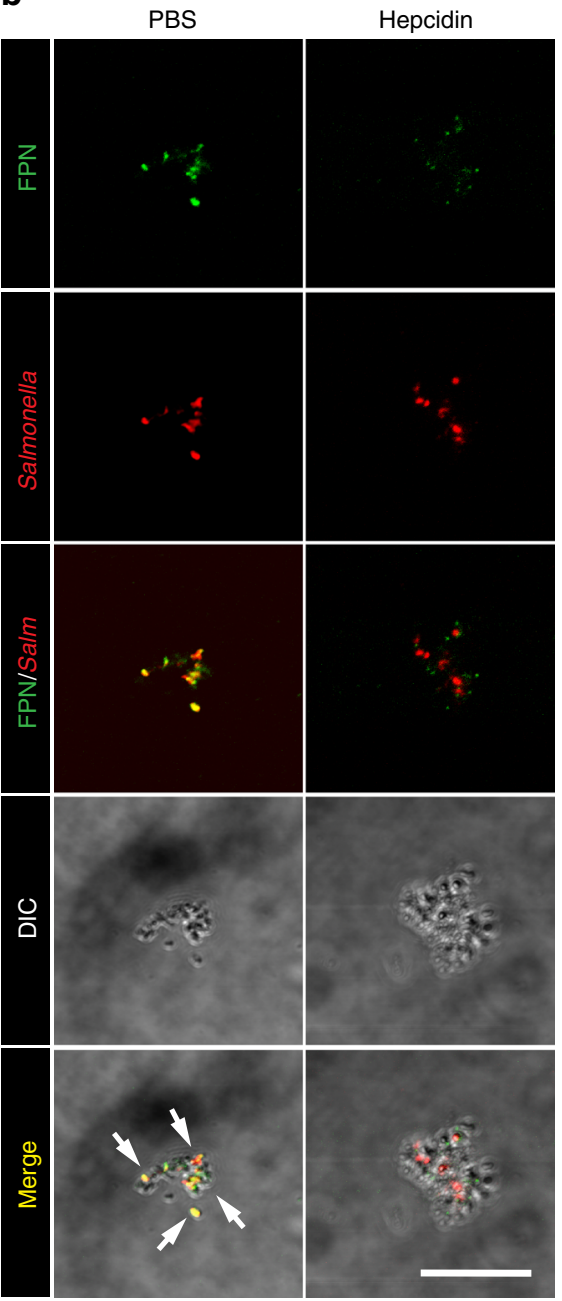

d

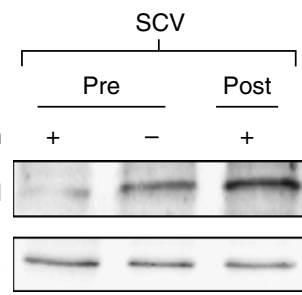

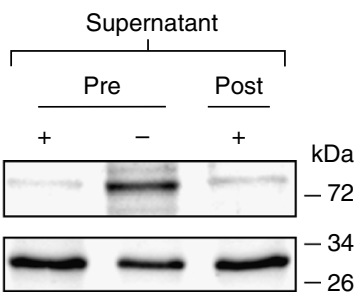

Fig. 1 The presence of FPN in the SCV of Raw264.7 cells. a Raw264.7 cells pretreated with $\mathrm{FeSO}_{4}(0.1 \mathrm{mM})$ and/or hepcidin $(1 \mu \mathrm{g} / \mathrm{ml}) \mathrm{for} 3 \mathrm{~h}$ were infected with Salmonella (MOI, 100) and examined by confocal microscopy. Enlarged images of merge panels are shown in Supplementary Fig. 17. b The cells pretreated with PBS or hepcidin were infected with magnetized Salmonella (MOI, 100) for $2 \mathrm{~h}$, and then SCV were isolated and Salmonella were analyzed by confocal microscopy. Staining for FPN is shown in green, and Salmonella in red. The isolated SCV were visualized by differential interphase contrast (DIC) imaging (scale bar $10 \mu \mathrm{m}$ ). Images are representatives of over 500 isolated SCVs per group from at least three independent experiments. c Raw264.7 cells pretreated with hepcidin were infected with magnetized Salmonella. FPN, Rab5, and annexin V in the SCV and the non-SCV fraction (supernatant) were examined by western blotting using specific antibodies. d Alternatively, Raw264.7 cells infected with Salmonella were treated with hepcidin for $2 \mathrm{~h}$ post infection and analyzed as in c 
macrophages and a 50-100-fold decrease in bacterial cell numbers in the liver and spleen, resulting in increased survival of the mice. This was interpreted as a typical representation of innate nutritional immunity, under the assumption that the iron status in the vacuoles in which Salmonella reside $(\mathrm{SCV})^{6}$ would be the same as in the macrophage cytosol.

In this study, we used a reporter system to assess iron and reactive oxygen species (ROS) levels in the SCV, and demonstrated that hepcidin affected ROS generation in macrophages by modulating iron levels in the SCV. This effect was dependent on the presence of FPN in the SCV.

\section{Results}

FPN associates with the SCV membrane in absence of hepcidin. Salmonella enters epithelial cells and macrophages by micropinocytosis and resides in unique membrane-bound compartments SCV 6 . Raw264.7 cells were pretreated with $\mathrm{FeSO}_{4}$ to induce FPN expression ${ }^{7}$ or with hepcidin to induce FPN degradation $^{3}$ and then infected with Salmonella for $2 \mathrm{~h}$ (Fig. 1a). Confocal microscopy confirmed an increase in FPN with $\mathrm{FeSO}_{4}$ treatment and a reduction in FPN with hepcidin treatment. Salmonella were located immediately adjacent to FPN in cells treated with $\mathrm{FeSO}_{4}$, suggesting that FPN is associated with the SCV (see a

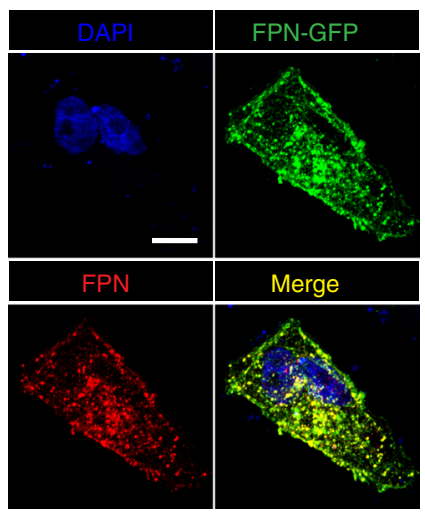

EEA1

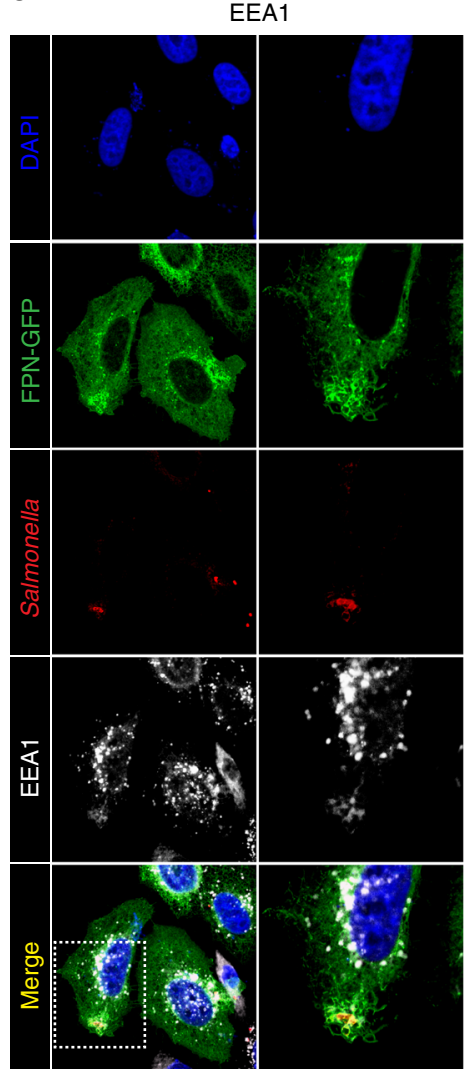

b

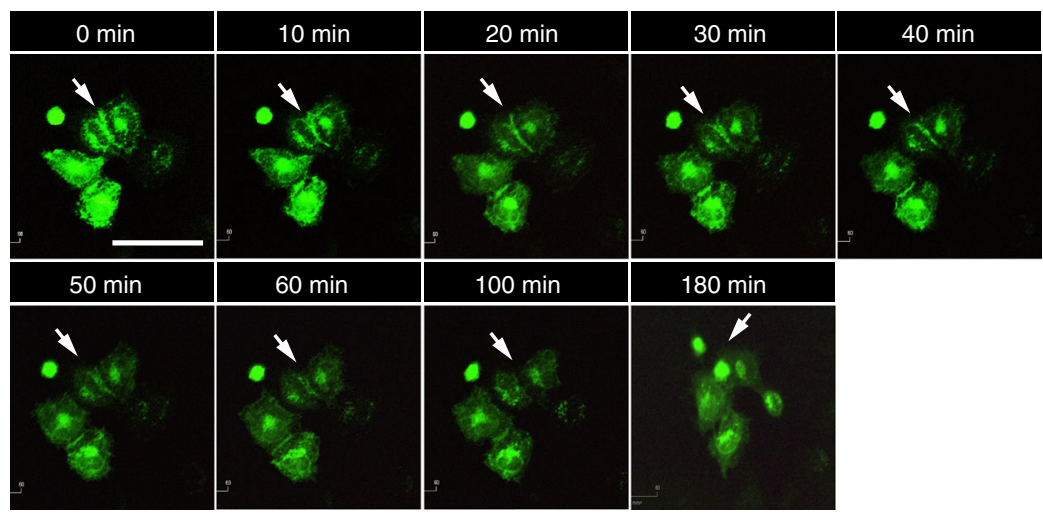

Rab5
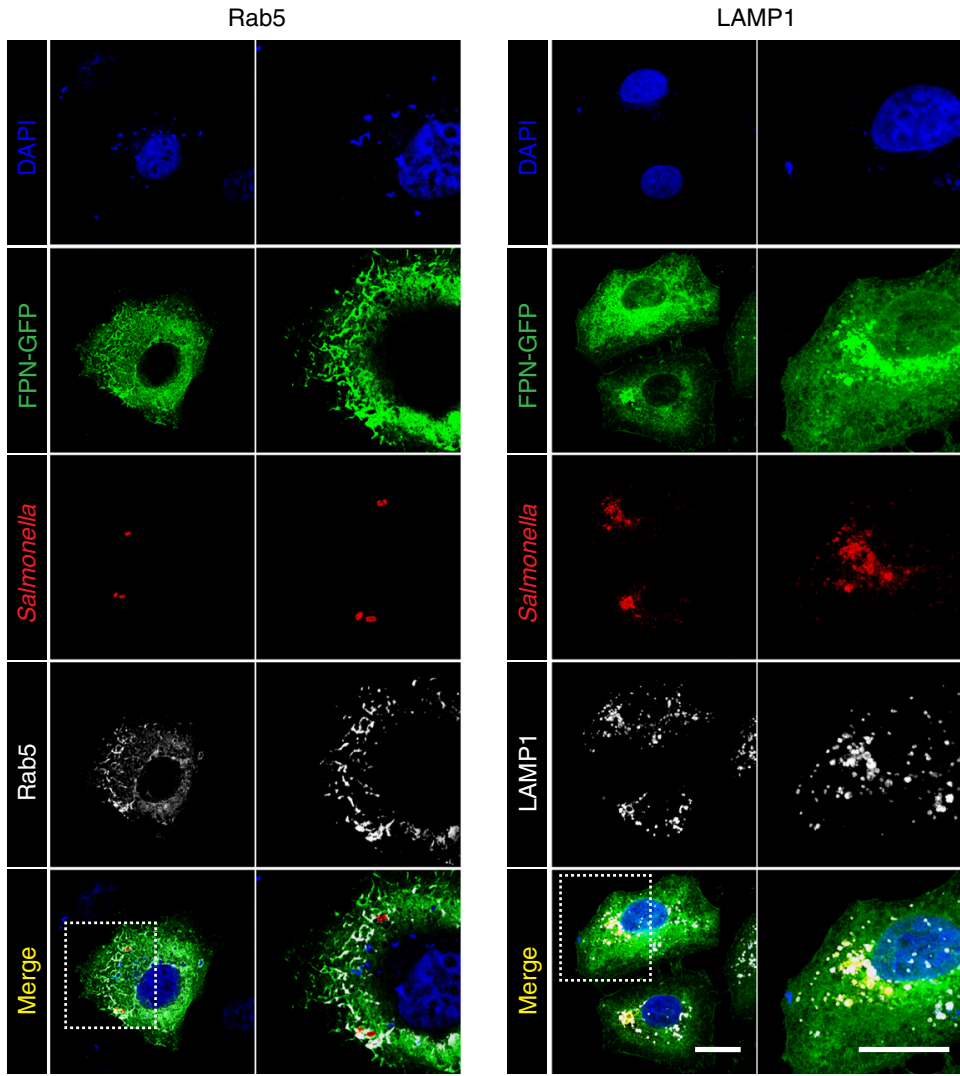

Fig. 2 Localization of FPN with respect to SCV-associated marker proteins. a HeLa cells transfected with pFPN-EGFP were stained with an anti-FPN antibody and visualized by confocal microscopy. Scale bar, $10 \mu \mathrm{m}$. b Live cell images after treatment of HeLa cells expressing FPN-GFP with hepcidin. Arrows indicate FPN on the plasma membrane. Scale bar, $50 \mu \mathrm{m}$. c HeLa cells expressing FPN-GFP were infected with Salmonella (MOI, 100) and stained for the markers of early and late SCV (EEA1 and Rab5 at $1 \mathrm{~h} \mathrm{p.i.} \mathrm{and} \mathrm{LAMP1} \mathrm{at} 12 \mathrm{~h}$ p.i.). Scale bar $10 \mu \mathrm{m}$. Images are representatives of over 100 infected cells from at least six independent experiments. The images on the left were taken at $\times 1000$ magnification and on the right at $\times 2000$ magnification, which were marked in dotted box on the left panel 
merged images). FPN remained with the SCV even at $12 \mathrm{~h}$ after infection in cells treated with $\mathrm{FeSO}_{4}$ (Supplementary Fig. 1). To further verify the above observation, SCV were isolated from phagocytic cells using magnetized bacteria ${ }^{8}$. Raw264.7 cells were pretreated with hepcidin, and then infected with Salmonella coated with magnetite nanoparticles, which does not alter total FPN levels in the cell (Supplementary Fig. 2). At $2 \mathrm{~h}$ post infection (p.i.), the cells were fractionated, and SCV containing magnetized a

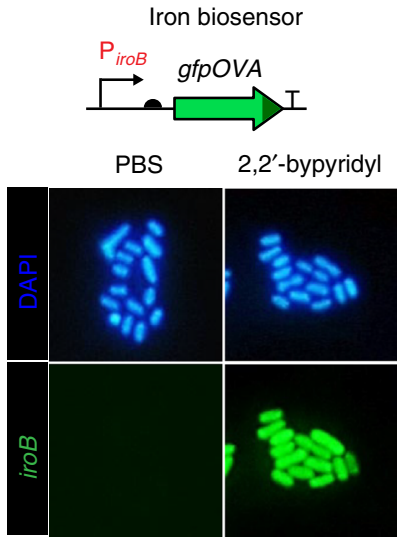

d

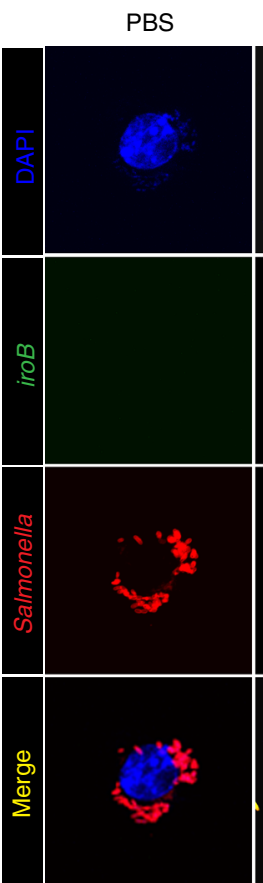

e

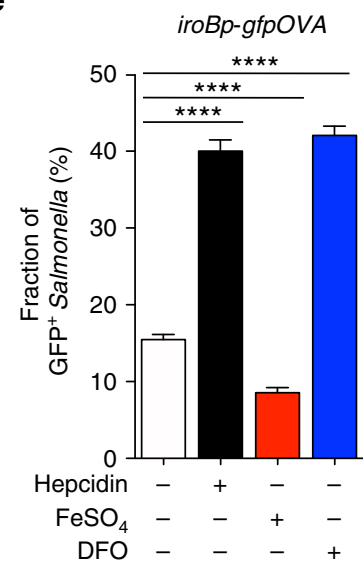

C
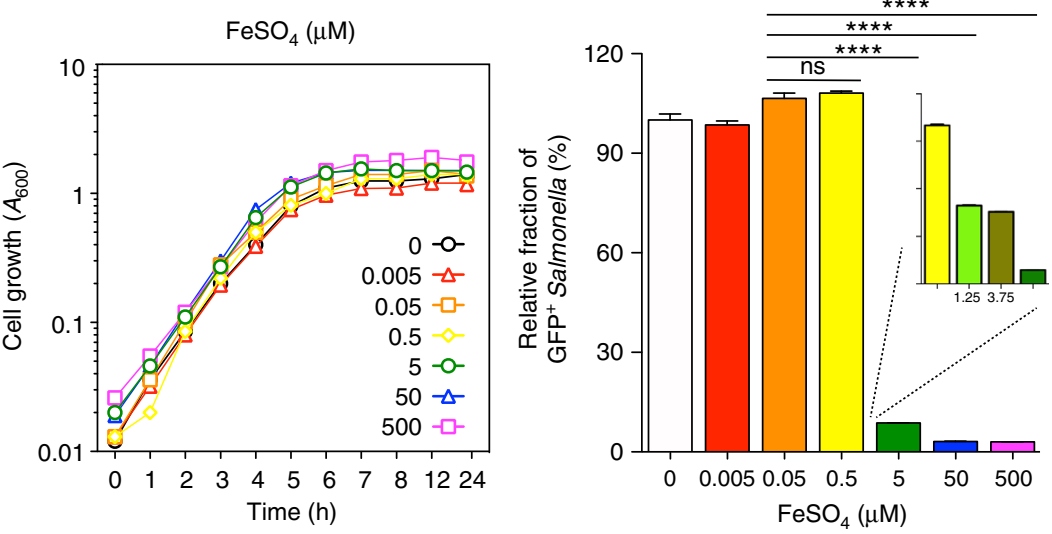

f

DFO
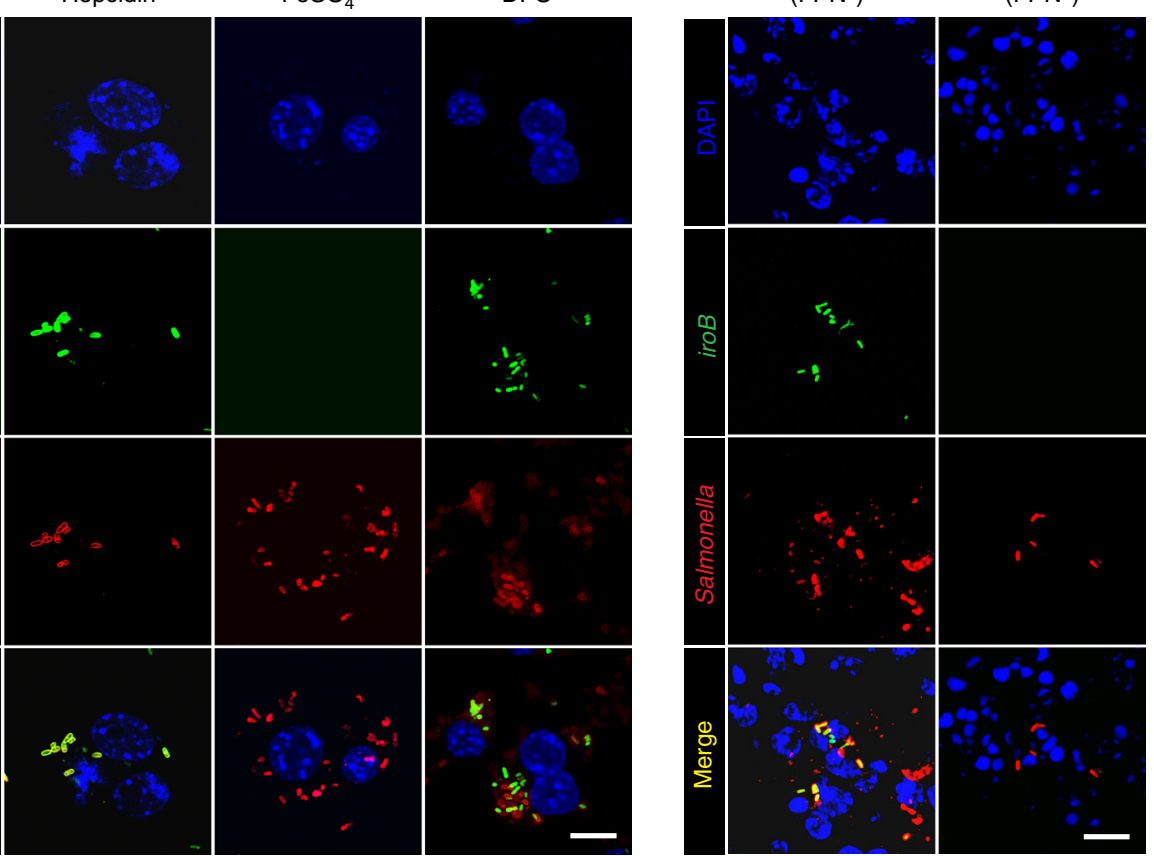

g

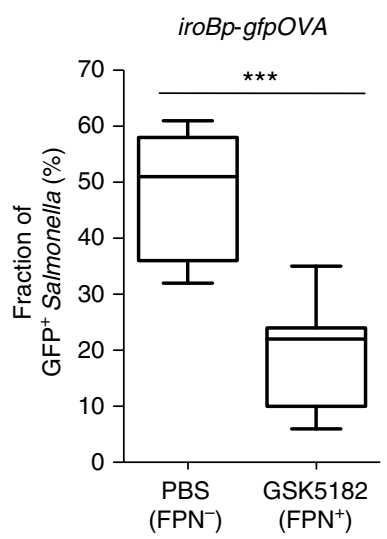


Salmonella were separated on a magnet. Initially, we analyzed the SCV fraction and the supernatant for the expression of the macrophage specific membrane marker, F4/80 ${ }^{9}$, and observed that it was only detected in the supernatant fraction, suggesting that the isolated SCV was free of most membrane components (Supplementary Fig. 3). We next probed the SCV for the presence of FPN and Salmonella by confocal microscopy using specific antibodies (Fig. 1b). FPN was detected only in the SCV isolated from PBS-treated cells, most of which appeared to encapsulate Salmonella. Without hepcidin treatment, FPN was detected by western blotting in the supernatant containing cellular debris and in the SCV fraction (Fig. 1c). When cells were pretreated with hepcidin, FPN was not detected in either fraction. Rab5 and MHC I, which are associated with SCV, as well as with the plasma membrane $e^{10-12}$, were detected in both fractions regardless of hepcidin treatment. Plasma membrane-bound annexin $\mathrm{V}^{13}$ was observed only in the supernatant fraction. These findings suggested that FPN is localized at or close to the SCV. To compare the effect of hepcidin before and after infection, Raw264.7 cells were infected with Salmonella for $1 \mathrm{~h}$ and then treated with hepcidin for an additional $1 \mathrm{~h}$. The fractions obtained from the samples post-treated with hepcidin showed FPN in the SCV but not in the cell membrane fraction contrasting the data obtained in hepcidin pretreated samples. Of note, hepcidin could not cross the plasma membrane to act on FPN in the SCV (Fig. 1d).

SCV undergo modifications directed by proteins encoded by Salmonella Pathogenicity Islands (SPIs). Initially, the SCV associates with markers of early endosomes, such as early embryonic antigen (EEA)1, the transferrin receptor, and Rab5. This is later followed by acquisition of other lysosomal markers, such as lysosome-associated membrane protein (LAMP)1. Thus, we attempted to localize FPN during SCV maturation with these markers using HeLa cells expressing a FPN-GFP fusion protein. HeLa cells were transfected with the $f p n-g f p$ fusion gene construct, and GFP expression was compared with that probed by specific anti-FPN antibody by confocal microscopy. Images of FPN-GFP showed a similar pattern of fluorescence as the images obtained with an anti-FPN antibody, suggesting that the GFP signal represented subcellular FPN localization (Fig. 2a). HeLa cells expressing FPN-GFP were next treated with hepcidin, and the GFP signal was evaluated by live cell imaging (Fig. 2b). Notably, the GFP signal at the cell membrane decreased in a timedependent manner, and was almost completely abolished by 100-180 min. A GFP signal in the cytosol would represent FPN being processed through the endoplasmic reticulum and the golgi apparatus for transportation to the plasma membrane. We therefore evaluated the co-localization of FPN with EEA1 and Rab5, early SCV markers, and LAMP1, a later SCV marker. FPNGFP localized immediately adjacent to EEA1 and Rab5 at $1 \mathrm{~h}$ p.i. and with LAMP1 at $12 \mathrm{~h}$ p.i., as observed by confocal microscopy (Fig. 2c). These were in marked contrast to the images of Salmonella-infected HeLa cells expressing GFP or uninfected
HeLa cells expressing FPN-GFP (Supplementary Fig. 4), where GFP signals were not found near the SCV-associated markers. Therefore, it appeared that FPN is associated with the SCV at all stages of maturation.

FPN acts as an iron transporter in the SCV membrane. We then investigated the role of FPN on the physiology of Salmonella within SCV with respect to iron availability. As direct measurement of iron content in the SCV is virtually impossible, a biosensor was utilized. The tandem iroN iroBCDE gene cluster on the Salmonella chromosome is involved in the uptake of catecholate-type siderophore compounds ${ }^{14}$. These genes are highly induced under iron-limited growth conditions and repressed under iron sufficiency by binding of the Ferric Uptake Regulator (Fur) $-\mathrm{Fe}^{2+}$ repressor complex to operator sites in the cognate promoter region (data not shown). The expression of these genes, therefore, corresponds to changes in the number of iron atoms per bacterial cell. We took advantage of this iron responsiveness to assess iron conditions within the SCV. First, the iron responsiveness of the $i r o B$ promoter (iroBp) was verified using Salmonella carrying a reporter plasmid (iroBp fused to lacZ) (Supplementary Fig. 5A). To measure the activity of this promoter in intra-macrophage Salmonella, another gene reporter system was constructed using the unstable GFP variant gfpOVA $^{15}$. The $g f p O V A$ was cloned downstream of iroBp on a pSC101-based plasmid ${ }^{16}$ that was introduced into Salmonella. Salmonella expressing gfpOVA were used to monitor iroBp activity with or without an iron chelator by determining the fraction of $\mathrm{GFP}^{+}$Salmonella (Fig. 3a). Quantification of the proportion of $\mathrm{GFP}^{+}$Salmonella revealed that iron chelation increased iroBp activity about 20-fold (Supplementary Fig. 5B). The expression of the iro $B$ gene transcript was quantified by qPCR and was also found to increase $\sim 20$-fold (Supplementary Fig. 5C). It is noteworthy that the expression of iroB was unaltered in response to other environmental changes such as magnesium concentration or $\mathrm{pH}$ shift (Supplementary Fig. 6). Taken together, these data demonstrate that iroBp is iron-responsive and that the fluorescence of Salmonella carrying iroBp-gfpOVA accurately reflected the $\operatorname{iro} B$ mRNA expression in an iron leveldependent manner. To determine the critical iron concentration for the modulation of $i r o B$ expression, Salmonella were grown in RPMI 1640, a chemically defined medium containing undetectable amounts of iron, supplemented with defined concentrations of $\mathrm{FeSO}_{4}(0.005-500 \mu \mathrm{M})$, and the iroBp activity was measured (Fig. 3b, c). Of note, Salmonella growth in the presence of increasing amounts of $\mathrm{FeSO}_{4}$ was indistinguishable, as reported previously (Fig. 3b $)^{17}$. Fluorescence from iroBp-gfpOVA was repressed at iron concentrations between 0.5 and $5 \mu \mathrm{M}$ in a concentration-dependent manner (Fig. 3c). The level of iroB mRNA determined by qPCR showed the same results (Supplementary Fig. 7). These data demonstrate that the iroBp-gfpOVA construct functioned accurately as an iron biosensor and that

Fig. 3 Assessment of SCV iron content in the presence or absence of FPN. a Expression of the iron biosensor in the presence or absence of iron chelator (2,2'-bypyridyl, $0.2 \mathrm{mM})$ in vitro. All Salmonella were stained with DAPI (blue), and iroBp-gfpOVA-expressing Salmonella are green. b Salmonella growth was determined in RPMI 1640 supplemented with the indicated concentration of $\mathrm{FeSO}_{4}(0.005-500 \mu \mathrm{M})$. Bacterial cell mass $\left(A_{600}\right)$ was measured at the indicated times. c Expression of iroBp-gfpOVA at $2 \mathrm{~h}$ p.i. in Salmonella grown as in $\mathbf{b}$ was quantified as the percentage of GFP+ Salmonella, as shown in $\mathbf{a}$. Data are presented as means \pm SEM. Significance is indicated as ${ }^{\star \star \star \star} p<0.0001$ by two-tailed Student's $t$-test. $\mathbf{d}$, $\mathbf{f}$ Nuclei were stained with DAPI (blue), Salmonella with a specific antibody (red), and iroBp-gfpOVA expression is shown in green. $\mathbf{d}$ Expression of iroB-gfpOVA was determined in the Salmonella (MOI, 100) infecting Raw264.7 cells pretreated with PBS, hepcidin $(1 \mu \mathrm{g} / \mathrm{ml})$, FeSO $4(0.1 \mathrm{mM})$, or DFO $(100 \mu \mathrm{M})$. Images were taken $2 \mathrm{~h}$ p.i. by confocal microscopy. $\mathbf{f}$ Expression of iroB-gfpOVA in the Salmonella infecting C57BL/6 mice treated with GSK5182 (FPN ${ }^{+}$) or PBS (FPN). Representative images of spleens infected with Salmonella were examined by confocal microscopy 2.5 days after i.v. infection $\left(1 \times 10^{5} \mathrm{CFU}\right)$. Scale bars $10 \mu \mathrm{m}$. e, $\mathbf{g}$ The fraction of iroBp-gfpOVA-expressing (green) Salmonella shown in $\mathbf{d}$, $\mathbf{f}$ was quantified, respectively, as in $\mathbf{b}$. Data are presented as means \pm SEM. Significance is indicated as ${ }^{\star \star \star} p=0.0004 ;{ }^{\star \star \star \star} p<0.0001$ by two-tailed Student's $t$-test 
Salmonella could grow in the presence of minute (nM) concentrations of iron.

Hepcidin-induced degradation of FPN on the macrophage plasma membrane resulted in an $\sim 30 \%$ increase in total cytosolic iron content (Supplementary Fig. 8). Changes in the iron level in the SCV in the presence or absence of FPN were assessed using Salmonella carrying the iroBp-gfpOVA biosensor. Raw264.7 cells cultured under iron overload conditions $\left(0.1 \mathrm{mM} \mathrm{FeSO}_{4}\right)$ or iron- a

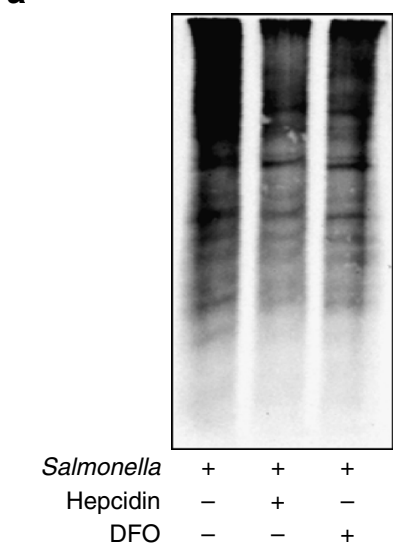

d

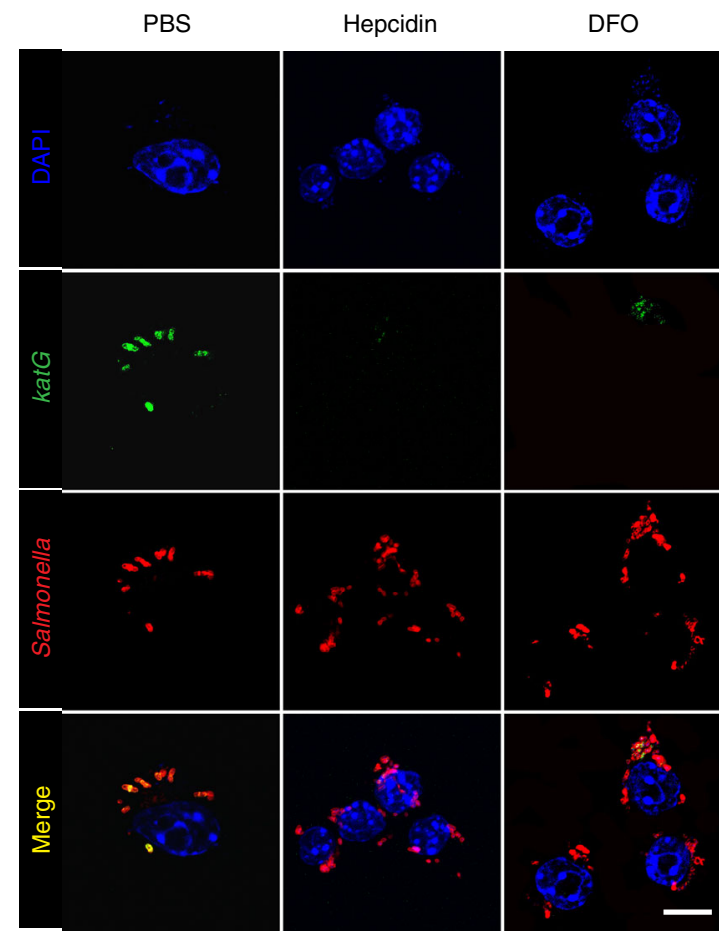

e

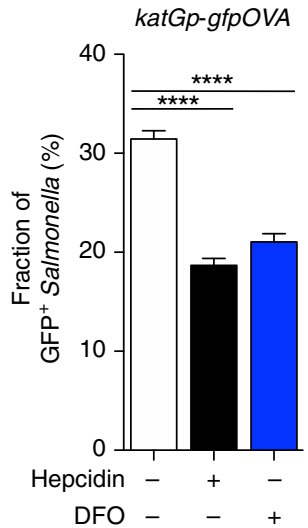

b
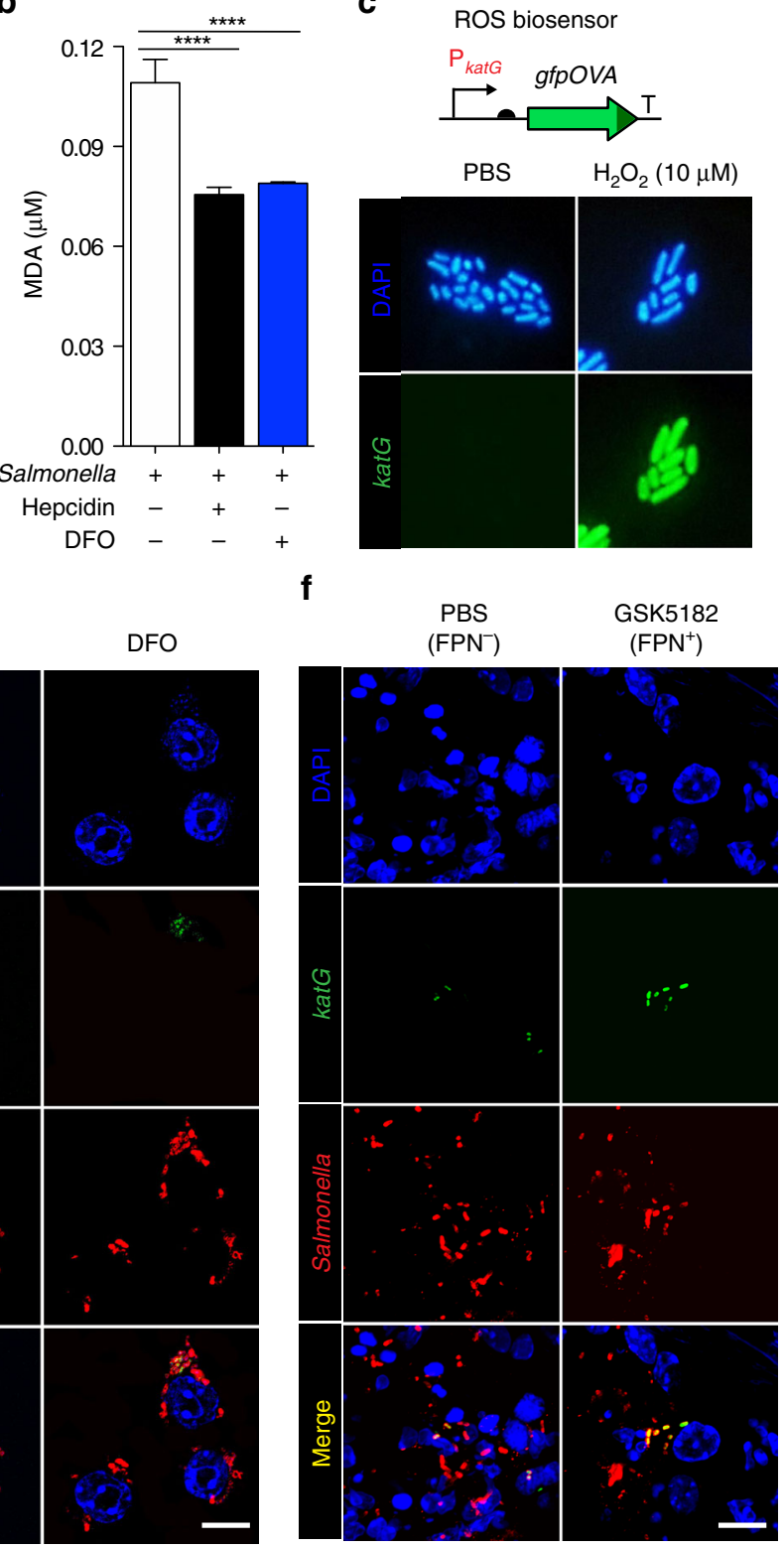

g

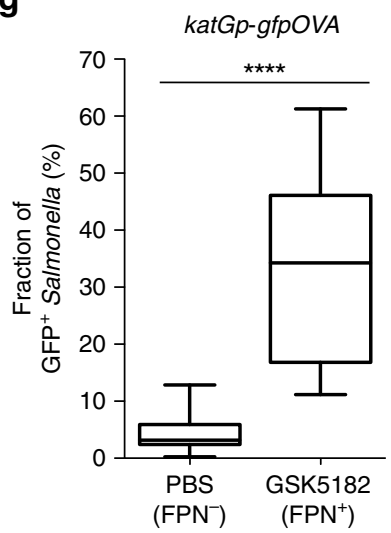


depleted conditions $100 \mu \mathrm{M}$ of the iron chelator deferoxamin $(\mathrm{DFO})^{18}$ were infected with Salmonella (MOI, 100), and iroBp activity was determined at $1 \mathrm{~h}$ p.i. (Fig. $3 \mathrm{~d}, \mathrm{e}$ ). The activity of iroBp, as determined by measuring the fraction of $\mathrm{GFP}^{+}$ Salmonella, increased in iron-depleted cells and decreased in iron-overloaded cells. Similar results were obtained when iroB mRNA levels were measured by qPCR (Supplementary Fig. 9). Most interestingly, depletion of FPN with hepcidin increased iroBp activity to the level observed in DFO-treated cells, suggesting that degradation of FPN reduced iron levels within the SCV in Raw264.7 cells. Upregulation of iroB with hepcidin was time-dependent, peaking at $2 \mathrm{~h}$ p.i. (Supplementary Fig. 10A). This indicates that FPN on the SCV acted as an iron transporter to move iron from the cytosol to the SCV, and depletion of FPN therefore resulted in iron-deficient conditions, leading to the activation of iroBp.

Finally, mice were infected with Salmonella carrying the iroBpgfpOVA biosensor and treated with GSK5182, which abrogates hepcidin expression and consequently allows FPN to be maintained. The iroBp activity of the Salmonella in the splenic macrophages was determined 2.5 days p.i. (Fig. 3f, g). Quantification of $\mathrm{GFP}^{+}$Salmonella (Fig. 3f) revealed that GSK5182 treatment repressed iroBp-gfpOVA expression by more than twofold (Fig. 3g). Downregulation of iroBp activity by GSK5182 treatment was confirmed by confocal microscopy, by counting the numbers of Salmonella (red) expressing GFP (green). Taken together, these data demonstrate that FPN on the SCV allows sufficient flow of iron into the SCV, resulting in downregulation of iroBp activity. By contrast, in the absence of FPN, the iron concentration in the SCV is reduced and iroBp activity is increased. FPN therefore acts as an iron transporter in the SCV membrane.

Decreased iron in the SCV impairs ROS-dependent killing. In the early stages of Salmonella infection, there is an abrupt increase in superoxide formation known as the oxidative burst, which is catalyzed by a NADPH oxidase enzyme complex, a prototypical hemoprotein complex with heme iron at the active site ${ }^{19-22}$. The superoxide is then converted to hydrogen peroxide, which is further converted to other highly reactive hydroxyl radicals by the iron-catalyzed Fenton reaction ${ }^{23,24}$. Since downregulation of hepcidin by GSK5182 in mice infected with Salmonella conferred on antimicrobial effect ${ }^{5}$, we investigated whether the hepcidin-FPN axis is involved in ROS generation in macrophages. Hallmarks of ROS-mediated cellular damage include protein carbonylation ${ }^{25}$ and lipid peroxidation ${ }^{26}$. These parameters were therefore assessed as means of evaluating ROS generation in the SCV. Raw264.7 cells pretreated with hepcidin or with DFO, which prevents ROS generation by inhibiting the Fenton reaction $^{27}$, were infected with magnetized Salmonella, and SCV were isolated $1 \mathrm{~h}$ p.i. SCV proteins were separated by SDSPAGE and probed for carbonylated moieties using specific antibodies (Fig. 4a). There was an $\sim 20 \%$ reduction in carbonylated proteins in SCV fraction from hepcidin-treated cells and an approximately $35 \%$ reduction in DFO-treated cells. Levels of malondialdehyde (MDA), the most abundant aldehyde produced by lipid peroxidation, were also measured by thiobarbituric acidreactive substances (TBARS) assay (Fig. 4b) ${ }^{26}$. Hepcidin and DFO reduced MDA levels similarly. These results suggest that hepcidin treatment inhibited ROS generation.

To further verify the effect of hepcidin on ROS generation, Salmonella carrying an episomal kat $G p$-gfpOVA fusion were used as a ROS biosensor ${ }^{16}$. The kat $G$ promoter is activated by the transcription factor OxyR upon exposure to $\mathrm{H}_{2} \mathrm{O}_{2}$ in a concentration-dependent manner (Fig. 4c) ${ }^{16,28}$. Raw264.7 cells pretreated with DFO or hepcidin were subsequently infected, and kat $G p$ activity was evaluated at $1 \mathrm{~h}$ p.i. The katGp activity in macrophages was determined by quantifying the fraction of $\mathrm{GFP}^{+}$Salmonella (Fig. 4d, e). Under DFO treatment, which results in low ROS generation, the fraction of $\mathrm{GFP}^{+}$bacteria was lower than in macrophages treated with PBS. Of note, treatment with hepcidin, which reduces FPN on the SCV, as well as on the plasma membrane, also reduced kat $G p$ activity. Similar results were observed when katG mRNA was measured by $\mathrm{qPCR}$ (Supplementary Fig. 11). The repressive effect of hepcidin on katGp activity was time-dependent (Supplementary Fig. 10B). Next, mice were infected with Salmonella expressing katGp$g f p O V A$ and treated with GSK5182, and the fraction of bacteria in the spleen expressing GFP was determined 2.5 days p.i. Confocal microscopy further revealed that GSK5182 treatment, which allows the maintenance of FPN, resulted in an $\sim 10$-fold increase in $\mathrm{GFP}^{+}$Salmonella (Fig. 4f, g). ROS generation by NADPH oxidase and the iron-catalyzed Fenton reaction is therefore modulated by hepcidin, which presumably limits the availability of the iron cofactor in the SCV by modulating the maintenance of FPN.

This model predicts that GSK5182 would not be effective in controlling Salmonella infection in mice deficient in nox2, which lack the gp91 component of NADPH oxidase, the main cellular source of $\operatorname{ROS}^{29}$. To test this hypothesis, wild-type (WT) and nox $2^{-/-}$mice were orally infected with Salmonella $\left(1 \times 10^{8}\right)$ and treated with GSK5182 or PBS, and the survival of infected animals was determined. In contrast to WT mice, GSK5182 did not have any therapeutic effect in the nox $2^{-1-}$ mice; in fact, nox $2^{-/-}$mice expired earlier than WT mice (Fig. 5a). The spleens of these mice were examined for FPN expression 2.5 days p.i. (Fig. 5b and Supplementary Fig. 12). FPN was expressed in the spleens of both WT and nox $2^{-/-}$mice treated with GSK5182. Apparently, GSK5182 abrogated hepcidin activity and allowed FPN to be maintained in splenic macrophages ${ }^{5}$. The bacterial loads of Salmonella in the spleen were determined by counting colony forming units (Fig. 5c). Consistent with the survival data (Fig. 5a), the bacterial load in WT mice was about half of that in the nox $2^{-/}$mice. However, although GSK5182 reduced Salmonella numbers in WT mice, GSK5182 had little effect on the bacterial load in nox $2^{-/-}$mice. Subsequently, peritoneal macrophages from WT and nox $2^{-1-}$ mice were isolated, pretreated with $\mathrm{DFO}, \mathrm{FeSO}_{4}$, or hepcidin, and infected with

Fig. 4 Assessment of ROS generation in SCV in the presence or absence of FPN. a Protein carbonylation in SCV of Raw264.7 cells treated with PBS, hepcidin, or DFO, as determined using 2,4-dinitrophenylhydrazine. SCV were isolated using magnetized Salmonella (MOI, 100) $2 \mathrm{~h}$ p.i. Proteins with 2,4dinitrophenylhydrazine groups were quantified using a specific antibody. b Lipid peroxidation was determined in cells treated as in $\mathbf{a}$. c-g ROS biosensor Salmonella (katGp-gfpOVA ${ }^{16}$ ) that fluoresce green in the presence of hydrogen peroxide were used to assess ROS levels in SCV. c Green fluorescence of katGp-gfpOVA-expressing Salmonella treated with $10 \mu \mathrm{M} \mathrm{H}_{2} \mathrm{O}_{2}$. d Expression of katGp-gfpOVA in the Salmonella infecting Raw264.7 cells pretreated with PBS, hepcidin $(1 \mu \mathrm{g} / \mathrm{ml})$, or DFO $(100 \mu \mathrm{M})$. Images were taken $2 \mathrm{~h}$ p.i. using a confocal microscope. f Expression of katGp-gfpOVA in the Salmonella infecting C57BL/6 mice treated with GSK5182 (FPN ${ }^{+}$) or PBS (FPN $\left.{ }^{-}\right)$. Spleens of mice infected with Salmonella i.v. ( $1 \times 10^{5}$ CFU) were examined by confocal microscopy 2.5 days p.i. d, f Nuclei were stained with DAPI (blue), Salmonella with a specific antibody (red), and katGp-gfpOVA expression is shown in green. Scale bars, $10 \mu \mathrm{m}$. e, $\mathbf{g}$ The fractions of biosensor-positive (green) cells were quantified as a percentage of the total intra-macrophage Salmonella (red) in the experiment shown in d, f. Data are presented as means \pm SEM. Significance is indicated as ${ }^{\star \star \star \star} p<0.0001$ by two-tailed Student's $t$-test 
Salmonella carrying the episomal iroBp-gfpOVA or katGpgfpOVA biosensors (Fig. 5d). The iroBp activity, which was determined using the fraction of GFP-expressing bacteria as a surrogate, increased in macrophages treated with hepcidin (FPN
-; high-iron level) and macrophages treated with $\mathrm{DFO}\left(\mathrm{FPN}^{+}\right.$; low-iron level), and decreased in macrophages pretreated with $\mathrm{FeSO}_{4}\left(\mathrm{FPN}^{+}\right.$; high-iron level). This was the case in macrophages

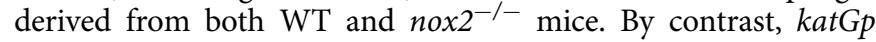

a

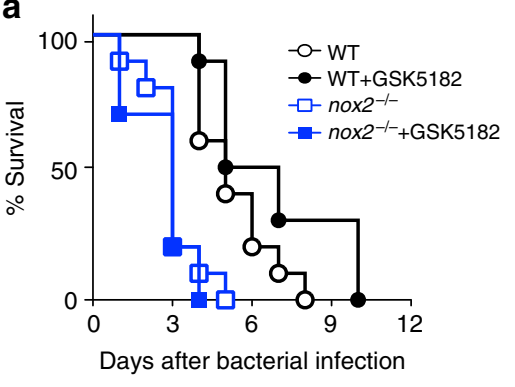

C

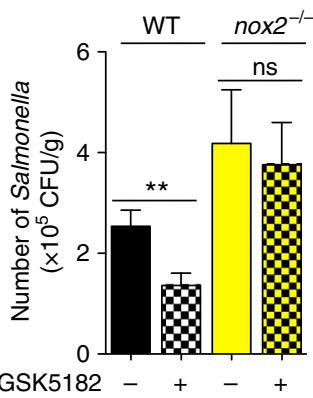

b

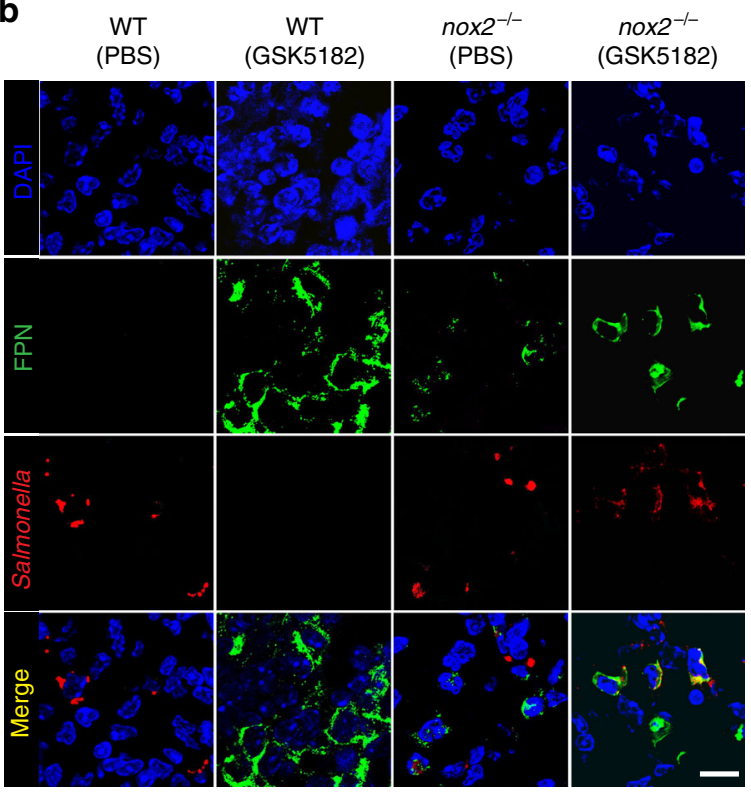

katGp-gfpOVA

d

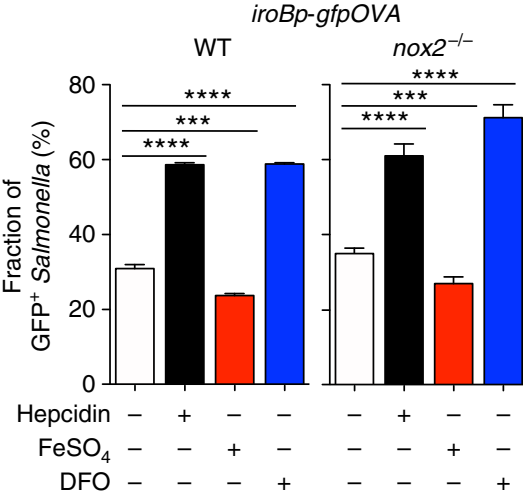

e

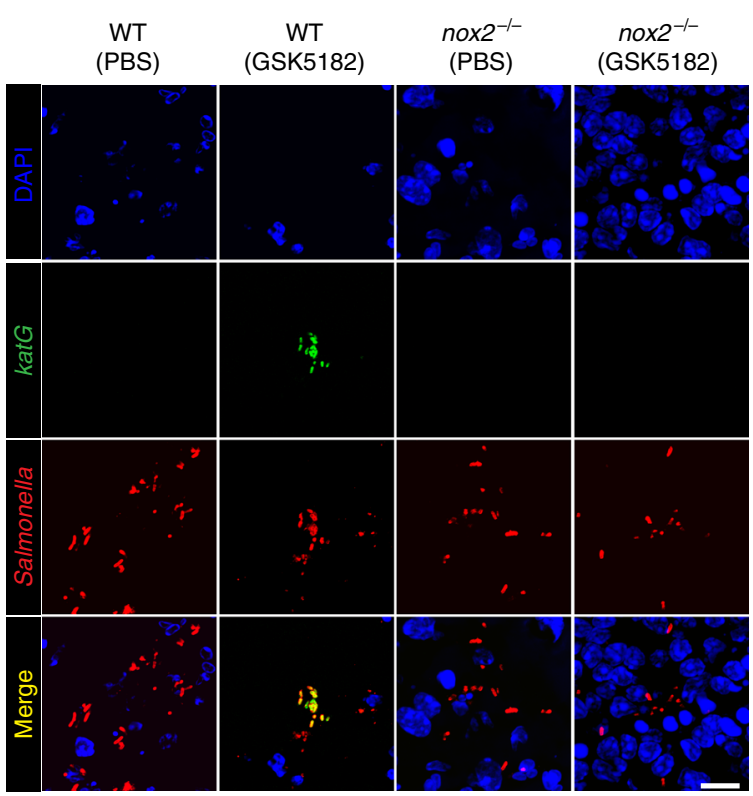

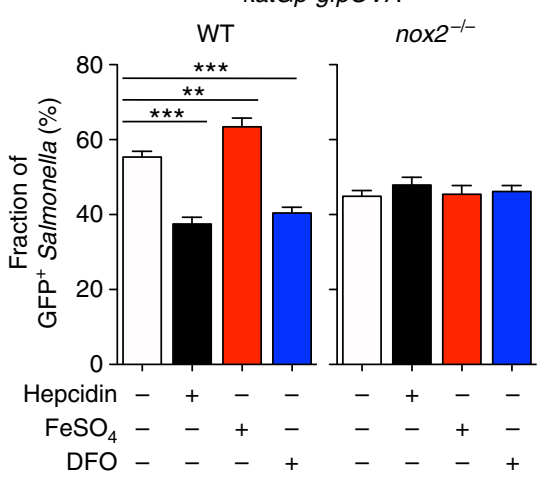

f

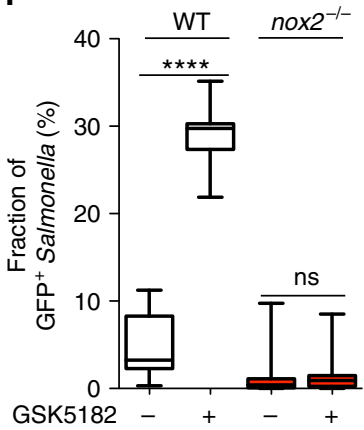


activity decreased in WT macrophages treated with hepcidin or DFO and increased in WT macrophages treated with $\mathrm{FeSO}_{4}$. Most interestingly, however, katGp activity in the nox $2^{-/-}$ macrophages did not change in response to hepcidin, DFO, or $\mathrm{FeSO}_{4}$, suggesting that hepcidin affected nox2-derived ROS. This finding was further verified using nox $2^{-/-}$mice. The effect of

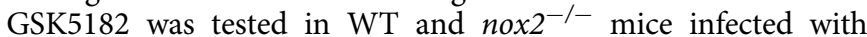
Salmonella expressing katGp-gfpOVA. The activity of katGp in the spleen was determined by quantifying the fraction of bacteria expressing $\mathrm{GFP}^{+}$Salmonella at 1.5 day p.i. (Fig. $5 \mathrm{e}$, f). In the absence of GSK5182, 71\% of Salmonella expressed GFP in WT mice, while only $15 \%$ of Salmonella in nox $2^{-1-}$ mice were $\mathrm{GFP}^{+}$. GSK5182 treatment further enhanced katGp activity in WT mice but not in $n o x 2^{-/-}$mice. Taken together, these findings indicate that the anti-Salmonella effect of GSK5182 treatment is dependent on ROS generation, which is affected by the presence or absence of FPN in the SCV.

Finally, WT mice were infected with Salmonella carrying iroBp-gfpOVA or katGp-gfpOVA and treated with GSK5182 or PBS. Whole spleens were isolated 2.5 days p.i. and examined for $\operatorname{iroB}$ or $k a t G$ expression by fluorescence microscopy (Fig. 6a and Supplementary Fig. 13). In PBS-treated mice, in which FPN is degraded, $i r o B$ was expressed but $k a t G$ was not. In GSK5182treated mice, in which FPN levels are maintained, iroB expression decreased while $k a t G$ expression increased. These results show that the maintenance of FPN leads to iron-sufficient conditions in the SCV for ROS generation (Fig. 6b).

\section{Discussion}

The initial stages of SCV formation and the composition of the SCV are only poorly understood. The evidence presented in this study suggests that FPN is selectively internalized along with Salmonella in macrophages, and transports iron into the SCV lumen as supported by the functional analyses (Figs. 1 and 3). FPN1 protein was also localized in M. tuberculosis phagosomes after infection of RAW264.7 macrophages ${ }^{30}$. In this case, however, the FPN1 was suggested to be involved in the export of iron from the phagosome into the cytosol. The Salmonella iron biosensor (iroBp-gfpOVA) was activated at below $\mu \mathrm{M}$ concentrations of iron and was repressed at higher levels. This suggests that iron levels in the SCV fluctuate in the $\mu \mathrm{M}$ range. It must be emphasized here that Salmonella grows normally when iron levels are in the $\mathrm{nM}$ range, presumably by producing the catecholate siderophores enterobactin and salmochelin ${ }^{31}$. The iron released from the hydrolyzed siderophores would then allow the survival and proliferation of intra-macrophage Salmonella. Thus, the concept of 'nutritional immunity' in the control of Salmonella must be used with caution, as Salmonella only require a minute quantity of iron for growth and also reside in a separate compartment within the macrophage. Thus, it is the presence or absence of FPN on the SCV membrane that determines iron levels in the SCV lumen: depletion of FPN increased cytosolic iron content but decreased the iron level in the SCV (Fig. 3 and Supplementary Fig. 8).

Generation of ROS is initiated by heme iron of flavocytochrome b558 in NADPH oxidase transferring electrons from NADPH to oxygen which is reduced to superoxide anion $23,24,32$. It is inferred that this activity of the NADPH oxidase must be functionally correlated with the availability of iron for insertion of heme prosthetic group into the apoprotein. Our results revealed that the iron level in SCV influenced ROS generation rather than Salmonella proliferation: Salmonella survived better in irondeficient conditions (no FPN) than in iron-sufficient conditions (with FPN) in vivo (Fig. 5). Studies with ROS biosensors revealed that hepcidin, which induces FPN degradation, reduced ROS generation in the SCV, as determined by measurement of oxidation of macromolecules, as well as katGp activity both in vitro and in a murine model (Fig. 4). A similar effect was reported in mice treated with DFO, which increased intracellular replication of Salmonella ${ }^{27}$. However, iron chelation has inhibitory effects on the replication of intra-macrophage Salmonella in iron starvation models based on another iron chelator, deferasirox ${ }^{33-35}$. We propose here that reduction of the available iron specifically in the SCV attenuates the production of hydroxyl radicals, which accounts for the capacity of these cells to limit intra-macrophage Salmonella growth ${ }^{36}$ (Supplementary Fig. 14). This model was based on the observation that depletion of FPN with hepcidin reduced iron intake into the SCV, which is needed for generation of antibacterial ROS in the SCV by NADPH oxidase and Fenton reaction (Fig. 4). Consequently, the increased survival of Salmonella-infected wild-type mice treated with GSK5182 was modest (Fig. 5a), possibly because ROS confer a critical antimicrobial effect early during the respiratory burst as Salmonella infect host cells anew ${ }^{37}$-GSK5182 removes only a part of various antimicrobial defenses in macrophages. It should be noted that irondependent ROS generation in SCV under this experimental condition was only $\sim 20 \%$ (Fig. $4 \mathrm{a}$, b), which might be sufficient to damage Salmonella without harming the organelles of the host cell. A similar ROS-dependent mechanism could account for the control of infection by intracellular bacteria such as Legionella pneumophila, and Mycobacterium tuberculosis and avium, known to reside in membrane-bound cytoplasmic vacuoles in macrophages $^{38-40}$. In addition, the iron transporter natural resistanceassociated macrophage protein 1 (Nramp1) was suggested to be involved in the regulation of iron level in SCV. Thus, mutations at Nramp1 cause susceptibility to infections by several intracellular pathogens, including Salmonella ${ }^{41-43}$. Therefore, we have executed the same set of experiments with peritoneal macrophages isolated from DBA2 mice $\left(\mathrm{Nrampl}^{+/+}\right)$to clarify the function of Nramp1 in regards to iron level in SCV. We observed similar patterns of iroB and $k a t G$ responses; iroB was increased and $k a t G$ was decreased following hepcidin treatment (Supplementary Fig. 15). Consistently, we have previously observed and reported a similar protective effect of GSK5182 in DBA2 mice $^{5}$. Taken together, this study provides a mechanism underlying the control

Fig. 5 Effect of nox2 mutation on Salmonella infection. a The survival of WT or nox $2^{-/-}$mice after oral infection with Salmonella $\left(5 \times 10^{8}\right)$ with or without GSK5182 treatment $(n=10)$. According to log-rank, Mantel-Cox survival test, only WT mice showed overall survival gain with GSK5182 treatment upon Salmonella infection $(p<0.002)$. b WT and nox $2^{-/-}$mice treated with PBS $\left(\mathrm{FPN}^{-}\right)$or GSK5182 $\left(\mathrm{FPN}^{+}\right)$were infected with Salmonella $\left(5 \times 10^{8} \mathrm{CFU}\right)$ orally. FPN and Salmonella in the spleen were examined by confocal microscopy 1.5 days p.i. using specific antibodies. Nuclei were stained with DAPI (blue), and Salmonella (red) and FPN (green) with specific antibodies. c Bacterial numbers (CFU/g) in the spleens of WT and nox2 $2^{-/-}$mice treated with GSK5182 were counted using the plating method. d Peritoneal macrophages were isolated from WT and nox $2^{-/-}$mice, treated with PBS, hepcidin $(1 \mu \mathrm{g} / \mathrm{ml})$, FeSO 4 $(0.1 \mathrm{mM})$, or DFO $(100 \mu \mathrm{M})$, and infected with Salmonella carrying episomal iroBp-gfpOVA or katGp-gfpOVA biosensors. The fraction of intra-macrophage Salmonella expressing gfpOVA was determined. e WT and nox $2^{-/-}$mice were infected orally with Salmonella carrying the episomal katGp-gfpOVA biosensor $\left(5 \times 10^{8}\right)$ and treated with PBS (FPN $\left.{ }^{+}\right)$or GSK51812 (FPN ${ }^{-}$). Spleens were examined by confocal microscopy 1.5 days p.i. Salmonella are shown in red and katGp-gfpOVA expression is shown in green. $\mathbf{f}$ The fraction of Salmonella expressing katGp-gfpOVA in the experiment shown in e. Data are presented as means \pm SEM. Significance is indicated as ${ }^{* \star \star} p<0.001 ;{ }^{\star \star \star \star} p<0.0001$ by two-tailed Student's t-test 
a
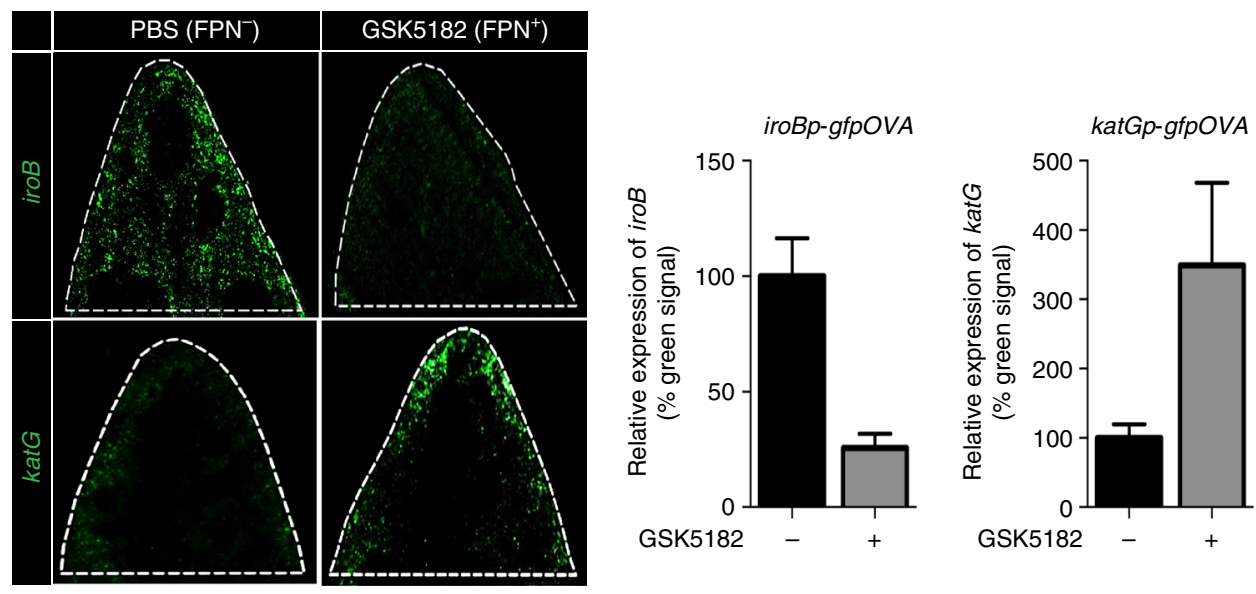

b

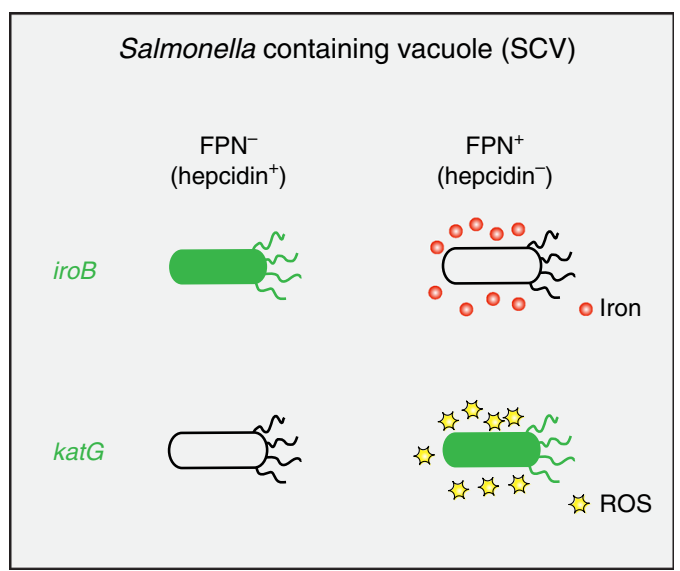

Fig. 6 Inverse correlation between iron level and ROS generation. a WT mice were infected with Salmonella carrying iroBp-gfpOVA or katGp-gfpOVA plasmid and treated with GSK5182 or PBS. Whole spleens were isolated 2.5 days p.i. and examined for iroB or kat $G$ expression by fluorescence microscopy. The relative expression of iroB or katG in the infected spleen shown in Supplementary Fig. 13 was determined by calculating relative intensity of green signal (\% $=$ intensity of green signal from GSK5182 treated/intensity of green signal from PBS treated) using ImageJ $1.41(\mathrm{NIH})$. Significance for iroB is $p=0.0091$; for katG, $p=0.0238$ by two-tailed Student's $t$-test. b Graphical summary

of intracellular bacterial infection by ERR $\gamma$ inverse agonist, GSK5182, although further studies would elucidate the role of Nramp1 in conjunction with FPN in intracellular bacterial infection.

\section{Methods}

Bacterial strains, plasmids and culture conditions. Salmonella typhimurium SL1344 was used as the WT strain. WT Salmonella and the pkatGp-gfpOVA plasmid were described previously ${ }^{16}$. The $i r o B$ reporter plasmid (piroBp-gfpOVA) was constructed by replacing the kat $G$ promoter of the pkatGp-gfpOVA plasmid with the $i r o B$ promoter region $(-200$ to +100$)$ using the Sph1 and Xbal restriction enzymes. The pFPN-EGFP plasmid was generated by ligating Xbal and EcoR1 fragments of full-length $f p n$ and pEGFP-N1 (Clontech). Bacterial cultures were grown in Luria broth (LB; Difco Laboratories) containing $1 \% \mathrm{NaCl}(\mathrm{w} / \mathrm{v})$ with vigorous aeration at $37^{\circ} \mathrm{C}$, and, when necessary, ampicillin (Sigma, \#A0166) was added at $100 \mu \mathrm{g} / \mathrm{ml}$, unless otherwise indicated. For solid support medium, Bacto agar (Difco Laboratories) was included at $1.5 \%(\mathrm{w} / \mathrm{v})$

Measurement of bacterial growth. Overnight bacterial cultures were diluted 1:100 into fresh $\mathrm{LB}$ and cultured for $3 \mathrm{~h}$ at $37^{\circ} \mathrm{C}$. The iron chelator $2,2^{\prime}$-bipyridyl (Sigma, \#D216305) was added, and bacteria were further cultured to test the impact of iron deprivation on bacterial growth. To examine the effect of iron concentration on bacterial growth, RPMI 1640 (Gibco/Thermo Fisher Scientific, \#1785905) was chosen as the culture medium. Overnight cultures were grown in RPMI 1640 medium containing $\mathrm{FeSO}_{4}(0.005-500 \mu \mathrm{M})$, and then diluted 1:100 into fresh medium containing the same concentration of iron and further incubated at $37^{\circ} \mathrm{C}$. Bacterial growth was measured every hour at an absorbance of $600 \mathrm{~nm}\left(A_{600}\right)$.
Culture of Raw264.7 macrophages, HeLa cells, and peritoneal macrophages RAW264.7 and HeLa cell lines were obtained from ATCC Korea and cultured according to the supplier's recommendation. Primary peritoneal macrophages were isolated from 8 -week-old C57BL/6 J mice (Jackson Laboratory) 3 days after elicitation by injection of $3 \%$ thioglycollate into the peritoneal cavity ( $1 \mathrm{ml}$ per mouse). The isolated macrophages were cultured in RPMI 1640 supplemented with $10 \%$ fetal bovine serum (FBS) in an incubator with $5 \% \mathrm{CO}_{2}{ }^{44}$. The macrophages were seeded at $5 \times 10^{5}$ cells per $\mathrm{ml}$ for all experiments.

Localization of FPN in HeLa cells upon Salmonella infection. HeLa cells were transiently transfected with pFPN-EGFP using FuGENE HD transfection reagent (Promega, \#E2311). Live cell images were taken of HeLa cells expressing FPN-GFP after treatment with hepcidin using the IncucyteS3 Live Cell Analysis System at $\times 20$ magnification (Essen Biosciences, USA). HeLa cells expressing FPN-GFP were stained with the following antibodies to SCV-associated marker proteins up to $12 \mathrm{~h}$ after Salmonella infection (MOI, 100): anti-EEA1 (Abcam, \#ab18211), anti-Rab5 (Abcam, \#ab2900), and anti-LAMP1 (Abcam, \#ab24170). Fluorescent signals were imaged with a LSM 880 confocal laser scanning microscopes equipped with VIS and NIR lasers. All captured images were taken using the Airyscan mode supported by the LSM 880 confocal laser scanning microscopy for image optimization (Carl Zeiss).

Measurement of gene expression using biosensors. Fluorescence of bacteria expressing the piroBp-gfpOVA was measured in vitro using SpectraFluor Plus (Tecan, Austria) at an excitation of $470 \mathrm{~nm}$ and an emission of $510 \mathrm{~nm}$. To measure the expression of $i r o B$ or kat $G$ by Salmonella within macrophages, Raw264.7 cells or peritoneal macrophages were infected with Salmonella expressing the piroBp-gfpOVA or pkatGp-gfpOVA, respectively. Infected macrophages were 
fixed at $1 \mathrm{~h}$ p.i. with $4 \%$ paraformaldehyde for $10 \mathrm{~min}$ at $4{ }^{\circ} \mathrm{C}$. The fixed cells were washed with PBS three times and then treated with ProLong Gold anti-fade reagent with 4',6-diamidino-2-phenylindole (DAPI; Invitrogen/Thermo Fisher Scientific, \#P36935). Salmonella were stained with an anti-Salmonella primary antibody (Abcam, \#ab8274) and an AlexaFluor 568-conjugated goat anti-mouse secondary antibody (Invitrogen/Thermo Fisher Scientific, \#A11031) (red). The stained cells were imaged on a fluorescence microscope and the expression of iroB or kat $G$ was quantified by comparison of the green and red signals in a single image using ImageJ $1.41(\mathrm{NIH})$. A total of 1712 images were analyzed.

Isolation of SCV. For isolation of SCV within Raw264.7 cells, magnetite particles (Bangs Laboratories, \#BM570) were used. The particles were prepared as described previously ${ }^{13}$. Briefly, the particles were washed twice with $0.1 \mathrm{M}$ MES buffer [2-( $N$ morpholino) ethanesulphonic acid, $\mathrm{pH}$ 5.2] using a magnetic rack after centrifugation of the stock suspension $(1 \mathrm{ml})$ at $1000 \times g$ for $30 \mathrm{~s}$ in a fixed-angle centrifuge. The washed particles were then resuspended with $4 \mathrm{mg} \mathrm{ml}^{-1}$ EDAC [1ethyl-3-(3-dimethylaminopropyl) carbodiimide], and mixed on a rotator (12 r.p. $\mathrm{m}$.) for $15 \mathrm{~min}$ at RT. The activated particles were washed twice with PBS (pH 7.4) using magnetic separation. The prepared particles were mixed with $0.5 \times 10^{9}$ live Salmonella and incubated at $37^{\circ} \mathrm{C}$ on a rotator (12 r.p.m.) for $30 \mathrm{~min}$. Raw264.7 cells were infected with the magnetized Salmonella at a MOI of 100. After infection, the cells were washed two times with DPBS (Well Gene, \#LB001-02) containing phenylmethanesulfonyl fluoride (PMSF) (10 mM; Sigma, \#P7626), and then treated with enzyme-free cell dissociation solution (Millipore, $\# \mathrm{~S}-004-\mathrm{B}$ ) in a $5 \% \mathrm{CO}_{2}$ incubator at $37^{\circ} \mathrm{C}$ for $2 \mathrm{~min}$. To inactivate the dissociation solution, RPMI containing PMSF $(10 \mathrm{mM})$ was added, and then the detached cells were transferred into a microfuge tube placed on a magnetic rack (Bioneer, MagListo-2, TM-1010). After washing with DPBS containing PMSF, the cells were fragmented with a Dounce homogenizer (Wheaton, \#357542), and then with a Polytoron homogenizer (IKA, Ultra-Turrax T 10), and then sonicated (Sonics, Vibra Cell VC505/ VC750). Finally, purified SCV were isolated using a magnetic rack. The SCV were analyzed by confocal microscopy or lysed by mixing with RIPA buffer (Bio-solution, \#BR002) containing a cocktail of proteinase $\mathrm{K}$ and protein phosphatase inhibitors (Thermos, \#RB228066, \#RA229831) on ice for western blot analysis.

Western blot analysis of FPN. Macrophages pretreated with hepcidin or PBS for $3 \mathrm{~h}$ were infected with Salmonella. The SCV were isolated from whole-cell lysates of macrophages as described above, and the remaining fraction (non-SCV fraction: supernatant) was analyzed by western blot. The samples $(50 \mu \mathrm{g})$ were run on $10 \%$ SDS- polyacrylamide gels and transferred to PVDF membranes (Amersham, $\# 10600002$ ). Primary antibodies were diluted 1:3000 in TBST (TBS with $0.2 \%$ Tween-20) and incubated for $16 \mathrm{~h}$ at $4{ }^{\circ} \mathrm{C}$. The following primary antibodies were used: anti-FPN (Novus Biologicals, \#NBP1-21502), anti-Rab5 (Abcam, \#ab18211), anti-annexin V (Abcam, \#ab14196), and anti-MHC class I (Novus Biologicals, \#NB120-15680). After washing with TBST three times, membranes were incubated with a 1:3000 dilution of horseradish peroxidase (HRP)-conjugated anti-rabbit (Thermo Fisher Scientific, \#31430) or anti-rat (Thermo Fisher Scientific, \#31470) secondary antibodies in TBST for $1 \mathrm{~h}$ at RT. After washing, the signals were visualized using chemiluminescence (Pierce, \#32106) and the Fusion Solo (Vilaber) imaging system. Uncropped images of blots are shown in Supplementary Fig. 16.

Analysis of ROS-related damage. The level of protein carbonylation was determined using the oxidized protein detection kit (Millipore, \#S7150-Kit). The lysed SCV were separated by SDS-PAGE and blotted on PVDF membranes, followed by western blot analysis ${ }^{45,46}$. In addition, ROS-related lipid peroxidation was estimated by thiobarbituric acid (TBA)-MDA assay using the TBARS assay kit (R\&D Systems, \#KGE013). The amount of lipid peroxidation in the sample was quantified by measuring TBARS levels at $532 \mathrm{~nm}$ using a SpectraFluor Plus instlument ${ }^{26}$.

Animal experiments. Male and female 8-week-old C57BL/6J WT and nox $2^{-/-}$ mice (B6.129S-Cybb $b^{t m 1 D i n} / \mathrm{J}$, stock number: 002365) were from the Jackson Laboratory (Bar Harbor, USA). The hepcidin inhibitor GSK5182 was synthesized as described previously ${ }^{5}$. To investigate the effect of GSK5182, mice were injected with PBS or GSK5182 $\left(40 \mathrm{mg} \mathrm{kg}^{-1}\right)$ intraperitoneally $12 \mathrm{~h}$ before Salmonella infection ( $n=5$ per group). After bacterial infection, mice were injected with PBS or GSK5182 once daily. Mice were infected with $1 \times 10^{8} \mathrm{CFU}$ of Salmonella orally or with $1 \times 10^{5} \mathrm{CFU}$ of Salmonella i.v. To determine the viable counts of Salmonella, spleens from five mice per group were collected and homogenized in sterile PBS containing $0.05 \%$ Tween-20 with a Polytoron homogenizer (IKA, UltraTurrax T 10). Salmonella was recovered from the homogenates and quantified by plating on agar. For the survival assay, $20 \mathrm{C} 57 \mathrm{BL} / 6 \mathrm{~J} \mathrm{WT}$ or $n o x 2^{-/-}$mice were infected orally with $1 \times 10^{8}$ Salmonella with or without GSK5182 treatment $(n=10$ per group). Survival rates were estimated by the Gehan-Breslow-Wilcoxon test. All animal experiments were approved and performed according to guidline of the Institutional Animal Use and Care Committee of the Chonnam National University (CNU IACUC-H-2016-15). For measurement of the expression of kat $G$ or iroB, mice were infected i.v. with $1 \times 10^{5} \mathrm{CFU}$ of Salmonella carrying the pkat $\mathrm{Cp}$ $g f p O V A$ or piroBp-gfpOVA plasmid, respectively. Gene expression was evaluated by analysis of red and green fluorescence using ImageJ, as described above.
Immunofluorescence staining and confocal microscopy. Raw264.7 cells were grown on sterilized glass cover slips for $12 \mathrm{~h}$. Pretreatments and Salmonella infection were carried out as described above. The cells were then fixed with $4 \%$ paraformaldehyde and blocked with $3 \%$ bovine serum albumin (BSA) in PBS. The samples were probed with a mouse anti-Salmonella antibody (1:200 in PBS Abcam, \#ab8274) and an AlexaFluor 568-conjugated secondary antibody (1:500 in PBS; Invitrogen/Thermo Fisher Scientific). The nuclei of the macrophages were stained with ProLong Gold anti-fade reagent with DAPI (Invitrogen/Thermo Fisher Scientific, P36935).

For in vivo experiments, the isolated spleens were fixed with $4 \%$ paraformaldehyde, embedded in optimal cutting temperature compound (OCT; Tissue-Tek), and frozen. The frozen spleens were sliced into $6-\mu \mathrm{m}$-thick sections using a microtome-cryostat. The tissue sections were mounted on aminopropyltriethoxysilane-coated slides. The slides were washed with PBS $(\mathrm{pH}$ 7.4) for complete removal of the OCT and incubated with primary antibodies [rabbit anti-mouse FPN (Alpha Diagnostic, \#MTP 11-A) and mouse antiSalmonella antibody (Abcam, \#ab8274)] at 1:200 in PBS for 2 days at $4{ }^{\circ} \mathrm{C}$. AlexaFluor 568-conjugated goat anti-mouse and AlexaFluor 488-conjugated goat anti-rabbit were used as secondary antibodies (1:100 in PBS). The samples were also stained with ProLong Gold anti-fade reagent with DAPI to detect nuclei. The stained images were captured with a Zeiss confocal microscope LSM 510 (Zeiss Laboratories) and the representative images were shown unless otherwise mentioned.

Statistical analysis. Data were analyzed using GraphPad Prism statistical software. The data met assumptions of a normal distribution as determined by statistical software, and variance was estimated using the standard error of the mean (SEM). The two-tailed Student's $t$-test was used to analyze differences between two groups. Differences were considered statistically significant at $p<0.05$.

Data availability. The data supporting the findings of the study are available in this article and its Supplementary Information files, or from the corresponding author upon request.

Received: 20 July 2017 Accepted: 27 April 2018

Published online: 29 May 2018

\section{References}

1. Hood, M. I. \& Skaar, E. P. Nutritional immunity: transition metals at the pathogen-host interface. Nat. Rev. Microbiol. 10, 525-537 (2012).

2. Nemeth, E. \& Ganz, T. The role of hepcidin in iron metabolism. Acta Haematol. 122, 78-86 (2009).

3. Nemeth, E. et al. IL-6 mediates hypoferremia of inflammation by inducing the synthesis of the iron regulatory hormone hepcidin. J. Clin. Invest. 113, 1271-1276 (2004)

4. Sajadi, M. M. \& Mackowiak, P. A. in Mandell, Douglas, and Bennett's Principles and Practice of Infectious Diseases. 8th edn (eds Bennett, J. E., Dolin, R. \& Blaser, M. J.) 708-720 (Saunders, USA, 2015).

5. Kim, D.-K. et al. Inverse agonist of estrogen-related receptor $\gamma$ controls Salmonella typhimurium infection by modulating host iron homeostasis. Nat. Med. 20, 419-424 (2014).

6. Ruiz-Albert, J. et al. Complementary activities of SseJ and SifA regulate dynamics of the Salmonella typhimuriumvacuolar membrane. Mol. Microbiol. 44, 645-661 (2002)

7. Liu, X., Hill, P. \& Haile, D. J. Role of the ferroportin iron-responsive element in iron and nitric oxide dependent gene regulation. Blood Cells Mol. Dis. 29, 315-326 (2002).

8. Wittrup, A. et al. Magnetic nanoparticle-based isolation of endocytic vesicles reveals a role of the heat shock protein GRP75 in macromolecular delivery. Proc. Natl. Acad. Sci. USA 107, 13342-13347 (2010).

9. Dos Anjos Cassado, A. F4/80 as a major macrophage marker: the case of the peritoneum and spleen. Results Probl. Cell Differ. 62, 161-179 (2017).

10. Feng, Y., Press, B. \& Wandinger-Ness, A. Rab 7: an important regulator of late endocytic membrane traffic. J. Cell Biol. 131, 1435-1452 (1995).

11. Garcia-del Portillo, F., Pucciarelli, M. G., Jefferies, W. A. \& Finlay, B. B. Salmonella typhimurium induces selective aggregation and internalization of host cell surface proteins during invasion of epithelial cells. J. Cell Sci. 107(Pt 7), 2005-2020 (1994).

12. Li, G. Rab5 GTPase and endocytosis. Biocell 20, 325-330 (1996).

13. Lönnbro, P., Nordenfelt, P. \& Tapper, H. Isolation of bacteria-containing phagosomes by magnetic selection. BMC Cell Biol. 9, 35 (2008).

14. Fischbach, M. A. et al. The pathogen-associated iroA gene cluster mediates bacterial evasion of lipocalin 2. Proc. Natl Acad. Sci. USA 103, 16502-16507 (2006). 
15. Rollenhagen, C., Sörensen, M., Rizos, K., Hurvitz, R. \& Bumann, D. Antigen selection based on expression levels during infection facilitates vaccine development for an intracellular pathogen. Proc. Natl. Acad. Sci. USA 101, 8739-8744 (2004).

16. Burton, N. A. et al. Disparate impact of oxidative host defenses determines the fate of Salmonella during systemic infection in mice. Cell. Host. Microbe 15, 72-83 (2014)

17. Kortman, G. A. M., Boleij, A., Swinkels, D. W. \& Tjalsma, H. Iron availability increases the pathogenic potential of Salmonella typhimurium and other enteric pathogens at the intestinal epithelial interface. PLoS ONE 7, e29968 (2012).

18. Paradkar, P. N. et al. Iron depletion limits intracellular bacterial growth in macrophages. Blood 112, 866-874 (2008)

19. Lieu, P. T., Heiskala, M., Peterson, P. A. \& Yang, Y. The roles of iron in health and disease. Mol. Asp. Med. 22, 1-87 (2001).

20. Liu, X. \& Zweier, J. L. A real-time electrochemical technique for measurement of cellular hydrogen peroxide generation and consumption: evaluation in human polymorphonuclear leukocytes. Free Radic. Biol. Med. 31, 894-901 (2001).

21. Lopes, L. R., Hoyal, C. R., Knaus, U. G. \& Babior, B. M. Activation of the leukocyte NADPH oxidase by protein kinase $\mathrm{C}$ in a partially recombinant cellfree system. J. Biol. Chem. 274, 15533-15537 (1999).

22. Mastroeni, P. et al. Antimicrobial actions of the NADPH phagocyte oxidase and inducible nitric oxide synthase in experimental salmonellosis. II. Effects on microbial proliferation and host survival in vivo. J. Exp. Med. 192, 237-248 (2000).

23. Cassat, J. E. \& Skaar, E. P. Iron in infection and immunity. Cell Host Microbe 13, 509-519 (2013).

24. Patruta, S. I. \& Hörl, W. H. Iron and infection. Kidney Int. Suppl. 69, S125-S130 (1999).

25. Moller, I. M. \& Kristensen, B. K. Protein oxidation in plant mitochondria as a stress indicator. Photochem. Photobiol. Sci. 3, 730-735 (2004).

26. Benzie, I. F. Lipid peroxidation: a review of causes, consequences, measurement and dietary influences. Int. J. Food Sci. Nutr. 47, 233-261 (1996).

27. Collins, H. L., Kaufmann, S. H. E. \& Schaible, U. E. Iron chelation via deferoxamine exacerbates experimental salmonellosis via inhibition of the nicotinamide adenine dinucleotide phosphate oxidase-dependent respiratory burst. J. Immunol. 168, 3458-3463 (2002).

28. Dubbs, J. M. \& Mongkolsuk, S. Peroxide-sensing transcriptional regulators in bacteria. J. Bacteriol. 194, 5495-5503 (2012).

29. Pollock, J. D. et al. Mouse model of X-linked chronic granulomatous disease, an inherited defect in phagocyte superoxide production. Nat. Genet. 9, 202-209 (1995).

30. Van Zandt, K. E. et al. The iron export protein ferroportin 1 is differentially expressed in mouse macrophage populations and is present in the mycobacterial-containing phagosome. J. Leukoc. Biol. 84, 689-700 (2008).

31. Hantke, K. Is the bacterial ferrous iron transporter FeoB a living fossil? Trends Microbiol. 11, 192-195 (2003).

32. Babior, B. M. NADPH oxidase. Curr. Opin. Immunol. 16, $42-47$ (2004).

33. Nairz, M. et al. Nitric oxide-mediated regulation of ferroportin-1 controls macrophage iron homeostasis and immune function in Salmonella infection. J. Exp. Med. 210, 855-873 (2013).

34. Nairz, M. et al. Iron regulatory proteins mediate host resistance to Salmonella infection. Cell Host Microbe 18, 254-261 (2015).

35. Nagy, T. A., Moreland, S. M., Andrews-Polymenis, H. \& Detweiler, C. S. The ferric enterobactin transporter Fep is required for persistent Salmonella enterica serovar Typhimurium infection. Infect. Immun. 81, 4063-4070 (2013).

36. Wenisch, C., Fladerer, P., Patruta, S., Krause, R. \& Hörl, W. Assessment of neutrophil function in patients with septic shock: comparison of methods. Clin. Diagn. Lab. Immunol. 8, 178-180 (2001)

37. Horwitz, M. A. \& Silverstein, S. C. Legionnaires' disease bacterium (Legionella pneumophila) multiples intracellularly in human monocytes. J. Clin. Invest. 66, 441-450 (1980).

38. Jabado, N. et al. Natural resistance to intracellular infections: natural resistance-associated macrophage protein 1 (Nramp1) functions as a $\mathrm{pH}$ dependent manganese transporter at the phagosomal membrane. J. Exp. Med. 192, 1237-1248 (2000).

39. Vidal, S. et al. The Ity/Lsh/Bcg locus: natural resistance to infection with intracellular parasites is abrogated by disruption of the Nramp1 gene. J. Exp. Med. 182, 655-666 (1995).
40. Canonne-Hergaux, F., Gruenheid, S., Govoni, G. \& Gros, P. The Nramp1 protein and its role in resistance to infection and macrophage function. Proc Assoc. Am. Physicians 111, 283-289 (1999).

41. Kuhn, D. E., Baker, B. D., Lafuse, W. P. \& Zwilling, B. S. Differential iron transport into phagosomes isolated from the RAW264.7 macrophage cell lines transfected with Nramp1Gly169 or Nramp1Asp169. J. Leukoc. Biol. 66, 113-119 (1999).

42. Nairz, M. et al. Slc11a1 limits intracellular growth of Salmonella enterica sv. Typhimurium by promoting macrophage immune effector functions and impairing bacterial iron acquisition. Cell Microbiol. 11, 1365-1381 (2009).

43. Zhang, X., Goncalves, R. \& Mosser, D. M. The isolation and characterization of murine macrophages. Curr. Protoc. Immunol. https://doi.org/10.1002/ 0471142735.im1401s83 (2008).

44. Chua, S. et al. Intra-coronary administration of tacrolimus markedly attenuates infarct size and preserves heart function in porcine myocardial infarction. J. Inflamm. 9, 21 (2012).

45. Cunha, T. F. et al. Exercise training prevents oxidative stress and ubiquitinproteasome system overactivity and reverse skeletal muscle atrophy in heart failure. PLoS ONE 7, e41701 (2012).

46. Rice, K. M. et al. Chronic paracetamol treatment influences indices of reactive oxygen species accumulation in the aging Fischer 344 X Brown Norway rat aorta. Ann. Clin. Lab. Sci. 42, 152-161 (2012).

\section{Acknowledgements}

This work has been supported by the National Research Foundation of Korea grant funded by the Korea government (NRF-2017R1A2A1A17069809). J.-H.J was supported by the Basic Science Research Program (NRF-2017R1A6A3A04006167) and K.S.K by Basic Science Research Program (NRF-2017R1A6A3A11032659). T.-H.L was supported by the R\&D grants for KMPC (NRF-2014M3A9D5A01073658). H.-S.C was supported by the National Creative Research Initiatives Grant (20110018305) through the National Research Foundation of Korea (NRF) funded by the Korean government (Ministry of Science, ICT \& Future Planning). D.B. was supported by the Swiss National Foundation (310030_156818). M.U.M was funded by the DFG SFB 1036.

\section{Author contributions}

D.L., J.-H.J., H.-S.C., and H.E.C. designed the experiments. D.L. and K.S.K. performed most of the experiments. D.L., M.S., and H.E.C. wrote the manuscript. D.L., J.-H.J., O.M., H.-J.K., T.-H.L., J.I.K., J.-J.M., D.B., and M.U.M analyzed data and reviewed the paper.

\section{Additional information}

Supplementary Information accompanies this paper at https://doi.org/10.1038/s41467018-04446-8.

\section{Competing interests: The authors declare no competing interests.}

Reprints and permission information is available online at http://npg.nature.com/ reprintsandpermissions/

Publisher's note: Springer Nature remains neutral with regard to jurisdictional claims in published maps and institutional affiliations.

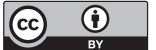

Open Access This article is licensed under a Creative Commons Attribution 4.0 International License, which permits use, sharing adaptation, distribution and reproduction in any medium or format, as long as you give appropriate credit to the original author(s) and the source, provide a link to the Creative Commons license, and indicate if changes were made. The images or other third party material in this article are included in the article's Creative Commons license, unless indicated otherwise in a credit line to the material. If material is not included in the article's Creative Commons license and your intended use is not permitted by statutory regulation or exceeds the permitted use, you will need to obtain permission directly from the copyright holder. To view a copy of this license, visit http://creativecommons.org/ licenses/by/4.0/.

(C) The Author(s) 2018 